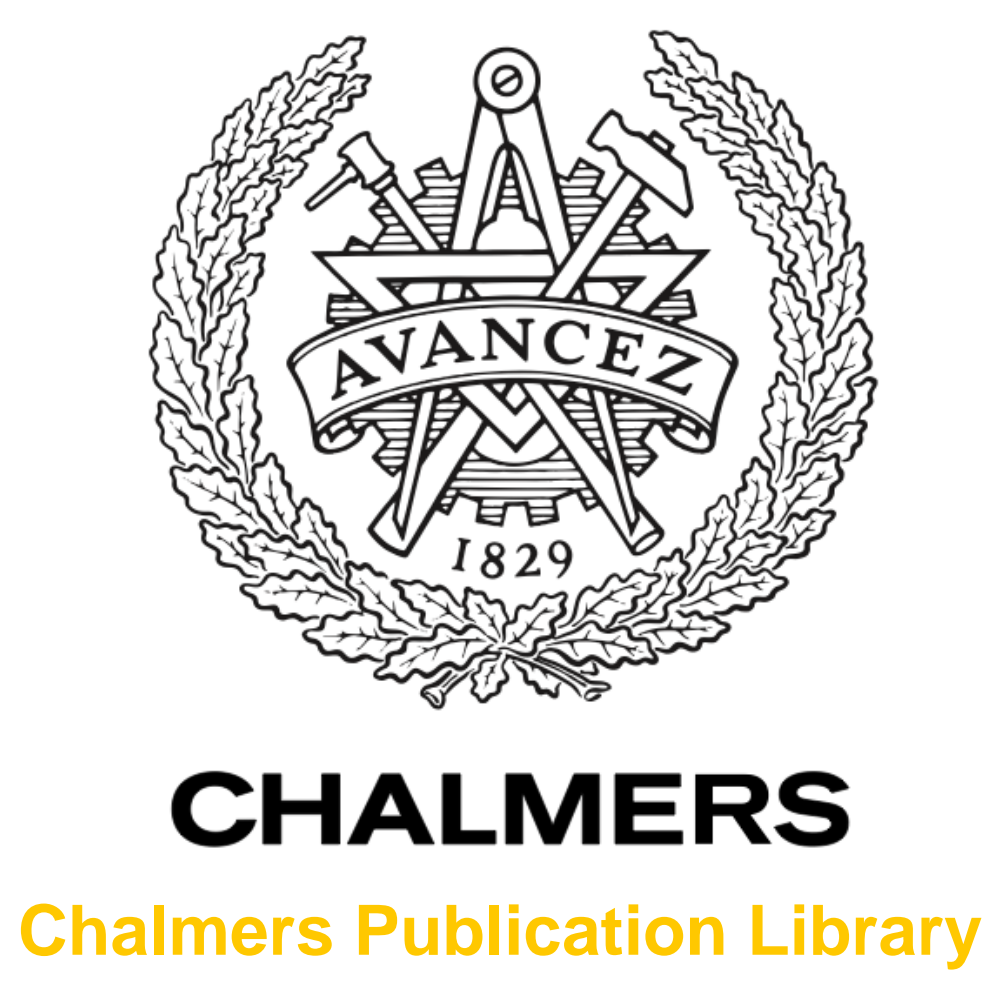

\title{
The effect of bias current asymmetry on the flux-flow steps in the grain boundary YBaCuO long Josephson junctions
}

This document has been downloaded from Chalmers Publication Library $(\mathrm{CPL})$. It is the author's version of a work that was accepted for publication in:

Journal of Applied Physics (ISSN: 0021-8979)

Citation for the published paper:

Revin, L. ; Chiginev, A. ; Pankratov, A. (2013) "The effect of bias current asymmetry on the flux-flow steps in the grain boundary $\mathrm{YBaCuO}$ long Josephson junctions". Journal of

Applied Physics, vol. 114(24), pp. 4.

http://dx.doi.org/10.1063/1.4856915

Downloaded from: http://publications.lib.chalmers.se/publication/193420

Notice: Changes introduced as a result of publishing processes such as copy-editing and formatting may not be reflected in this document. For a definitive version of this work, please refer to the published source. Please note that access to the published version might require a subscription. 


\title{
The effect of bias current asymmetry on the flux-flow steps in the grain boundary YBaCuO long Josephson junctions
}

\author{
L. S. Revin, ${ }^{1,2}$ A. V. Chiginev, ${ }^{1,2}$ A. L. Pankratov, ${ }^{1,2, a)}$ D. V. Masterov, ${ }^{1}$ A. E. Parafin, ${ }^{1}$ \\ G. A. Luchinin, ${ }^{3,1}$ E. A. Matrozova, ${ }^{2}$ and L. S. Kuzmin ${ }^{4,2}$ \\ ${ }^{1}$ Institute for Physics of Microstructures of RAS, GSP-105, Nizhny Novgorod 603950, Russia \\ ${ }^{2}$ Laboratory of Cryogenic Nanoelectronics, Nizhny Novgorod State Technical University, Nizhny Novgorod, \\ Russia \\ ${ }^{3}$ Institute of Applied Physics of RAS, Nizhny Novgorod 603950, Russia \\ ${ }^{4}$ Chalmers University of Technology, SE-41296 Gothenburg, Sweden
}

(Received 23 September 2013; accepted 9 December 2013; published online 26 December 2013)

\begin{abstract}
The current-voltage characteristics and critical current versus magnetic field dependence of long $24^{\circ}[001]$-tilt $\mathrm{YBa}_{2} \mathrm{Cu}_{3} \mathrm{O}_{7-\delta}$ bicrystal grain-boundary junctions are studied both experimentally and theoretically. For the opposite magnetic field directions, the flux-flow steps with significantly different height and slope are observed. It is demonstrated that the most probable reason of this discrepancy is recently predicted asymmetry of spatial bias current distribution due to crystallographic anisotropy of bicrystal substrates [Kupriyanov et al., JETP Lett. 95, 289 (2012)]. (C) 2013 AIP Publishing LLC. [http://dx.doi.org/10.1063/1.4856915]
\end{abstract}

\section{INTRODUCTION}

The investigation of asymmetric regimes arising in Josephson superconducting systems is of importance for various applications. An intriguing magnetic-field behavior of critical current $I_{c}(H)$ has attracted a considerable interest both in the low- $T_{c}{ }^{1-4}$ and high- $T_{c}^{5,6}$ Josephson junctions (JJs). Recently, it was demonstrated ${ }^{7}$ that the asymmetry of the magnetic differential pattern $I_{c}(H)$ in the inline low- $T_{c}$ Josephson junctions can be attributed to the different electrode inductances. The inhomogeneity of the critical current density in the case of high- $T_{c}$ superconducting materials with anisotropic pairing, like cuprate superconductors, is the result of the film growth on the bicrystal substrate. ${ }^{8,9}$ Unlike the artificial creation of asymmetric spatial bias current distribution in low- $T_{c}$ JJs using the unbiased tail for variation of the emitted power and the linewidth, ${ }^{10-14}$ the natural asymmetry of spatial current distribution in grain boundary junctions (GBJs) due to crystallographic anisotropy of bicrystal substrates has been recently predicted in Ref. 15 .

The regime of magnetic vortex flow is interesting both from fundamental and applied points of view, and was investigated experimentally ${ }^{16-19}$ and theoretically ${ }^{11-14,20-28}$ mostly for low-temperature Josephson junctions. For high-temperature JJs, there are only a few works on GBJs ${ }^{29-31}$ in this regime.

The aim of this paper is the experimental observation of the discrepancy of flux-flow (velocity-matching) steps for the opposite magnetic field directions in the $\mathrm{YBaCuO}$ GBJs and the discussion of the possible reasons leading to occurrence of such discrepancy.

\section{EXPERIMENTAL RESULTS}

The GBJs were fabricated by on-axis dc magnetron sputtering ${ }^{32}$ of $\mathrm{YBa}_{2} \mathrm{Cu}_{3} \mathrm{O}_{7-\delta}$ thin films on the surface of $24^{\circ}$ [001]-tilt $\mathrm{Zr}_{1-x} \mathrm{Y}_{x} \mathrm{O}_{2}$ bicrystal substrates (Fig. 1). The

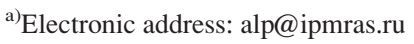

length of the junctions $l$ along the grain boundary was $350 \mu \mathrm{m}$ and the thickness was $0.3 \mu \mathrm{m}$. The samples were mounted into the pulse tube PT- 410 cryostat and characterized with the precise low-noise current source by standard 4-probe technique. The junctions had resistively shunted-junction like $I-V$ curves at temperatures up to $80 \mathrm{~K}$. The junctions were very long since their lengths are much larger than the Josephson penetration depth $\lambda_{J}=\sqrt{\Phi_{0} /\left(2 \pi \mu_{0} J_{c} d\right)}$, which determines the size of a fluxon in the junction. Here, $\Phi_{0}$ is the magnetic flux quantum, $\mu_{0}$ is the vacuum permeability, $J_{c}$ is the critical current density, and $d=t+2 \lambda_{L}$ is the effective magnetic thickness with the junction barrier thickness $t=1.5 \mathrm{~nm}$ and London penetration depth $\lambda_{L}=150 \mathrm{~nm}$. For a typical $J_{c}=50 \mathrm{kA} / \mathrm{cm}^{2}$ at $\sim 6 \mathrm{~K}$, the Josephson penetration depth was about $1.3 \mu \mathrm{m}$. The magnetic field perpendicular to the grain boundary was produced by a current $I_{C L}$ through the copper wire coil, having the inner diameter more than order of magnitude larger than the junction length. Since the junction was placed precisely into the coil center, it was assumed that the magnetic field along the junction was nearly uniform.

In the inset of Fig. 2, the dependence of critical current $I_{c}$ on the current through the coil $I_{C L}$ is presented. As is expected for a long grain boundary junction with the misorientation angle $24^{\circ}, I_{c}(H)$ dependence has some distortions and does not go down to zero with magnetic field increase, ${ }^{6,33}$ see also the second plot of Fig. 37 of Ref. 34 . The reasons are different defects, like high current paths, and also a flux creep.

Figure 2 shows the $I-V$ curves for various values of applied magnetic field. Here, $I$ is the bias current through the junction and $R_{N}$ is the normal state resistance. We observe a field dependent, resonant-like steps at the $I-V$ curves. We relate these steps to the flux-flow (velocity matching) steps ${ }^{29-31}$ since they scale approximately linear on the applied magnetic field, as it must. ${ }^{29}$ Due to high damping in a GBJ, these steps are much less steep than those in long 


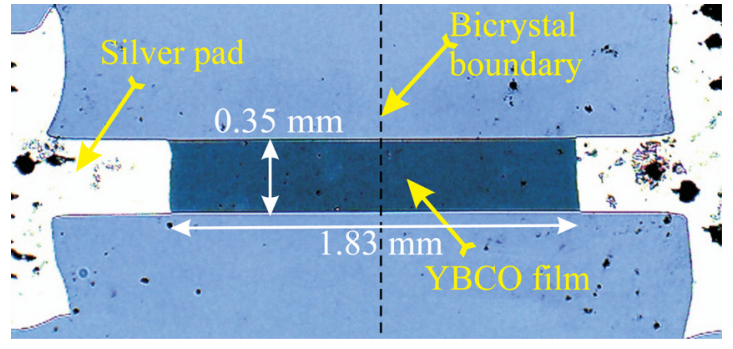

FIG. 1. The photo of the working sample. Black defects at silver pads are places of bonding.

$\mathrm{NbAlO}_{x} \mathrm{Nb}$ junctions. ${ }^{18}$ The steps are clearly visible in the voltage range below $2 I_{c} R_{N}$. In order to determine the steps accurately, we subtract the normal current, $V / R_{N}$, from the bias current $I$ (the right axis of Fig. 2). Here, $R_{N}=0.0087 \Omega$ $\left(I_{c} R_{N}=0.46 \mathrm{mV}\right.$ for $\left.T=6 \mathrm{~K}\right)$ was determined approximately for high bias currents and the dependence shown in the right axis of Fig. 2 considered only for convenience. For fields with the coil current $\left|I_{C L}\right|<1 \mathrm{~mA}$, only weak traces from the flux-flow steps can be seen because the density of vortices in the junction is low at such weak fields. The most clear resonant step is observed for $\left|I_{C L}\right|=2.25 \mathrm{~mA}$ with the minimal differential resistance $\mathrm{d} V / \mathrm{d} I$ at the top. At larger fields $\left|I_{C L}\right|>3 \mathrm{~mA}$ vortices tightly fill the junction and the differential resistance tends to the normal state resistance.

We have measured a similar dependence for the positive coil currents and found the sharper flux-flow step at the same modulo values of $I_{C L}=2.25 \mathrm{~mA}$. It is seen from Fig. 3 that for one magnetic field direction (negative coil current) the flux-flow (velocity-matching) step is sharper than for the other (positive coil current). Different direction of the magnetic field with the same sign of the bias current leads to the different direction of fluxons motion along the junction. Thus, for some reasons the regime of fluxon motion from one side to another occurring with the negative $I_{C L}$ values is

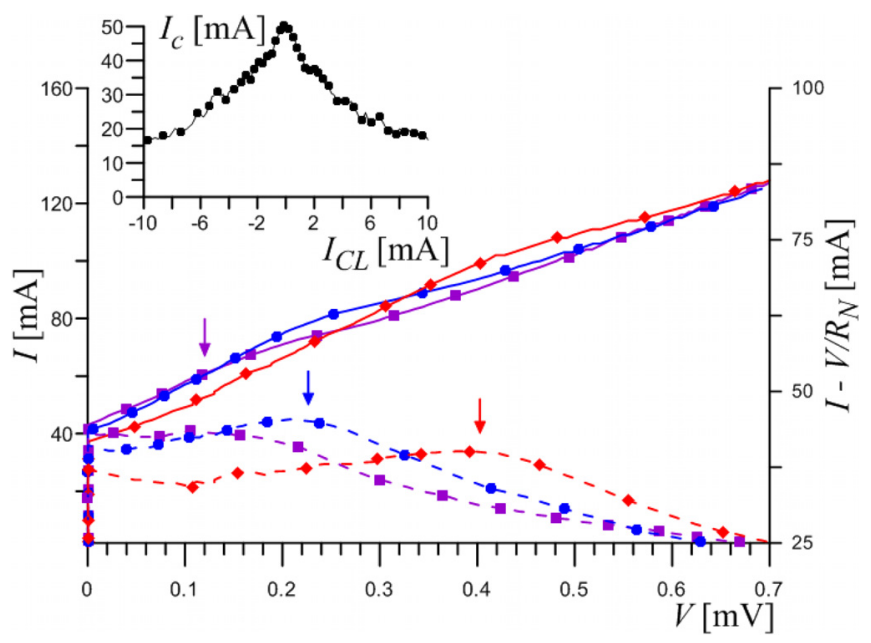

FIG. 2. Magnetic field response of GBJ at $T=6 \mathrm{~K}$. The $I$ vs $V$ (solid) as well as $\left(I-V / R_{N}\right)$ vs $V$ (dashed) curves correspond to increasing coil current $I_{C L}: I_{C L}=-1 \mathrm{~mA}$ (curve with rectangles), $I_{C L}=-2.25 \mathrm{~mA}$ (curve with circles), and $I_{C L}=-3 \mathrm{~mA}$ (curve with diamonds). The maximum heights of the flux-flow steps are indicated with arrows. The inset: the critical current versus the current applied to the coil at the temperature of $6 \mathrm{~K}$. Only a small fraction of measured points is shown by symbols.

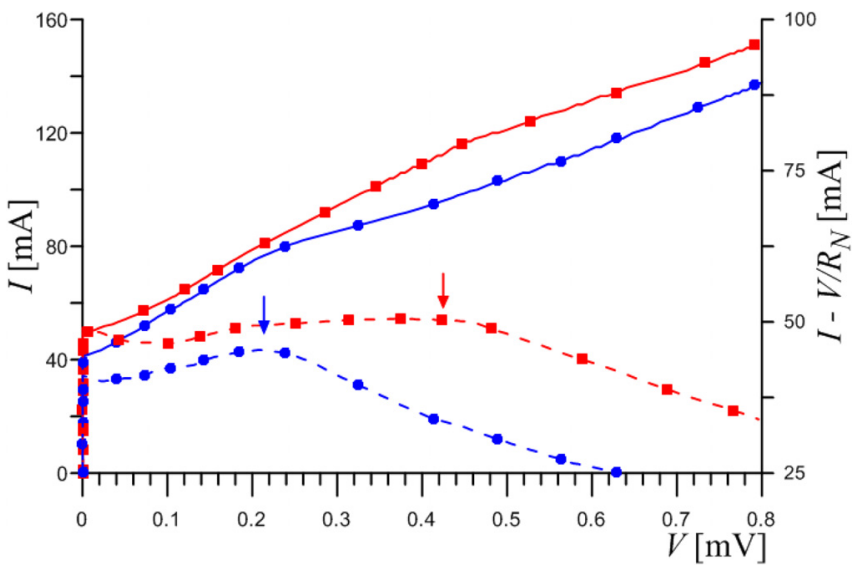

FIG. 3. Magnetic field response of GBJ at $T=6 \mathrm{~K}$. $I$ vs $V$ (solid) as well as $\left(I_{\text {bias }}-V / R_{N}\right)$ vs $V$ (dashed) curves correspond to the different magnetic field directions, i.e., for $I_{C L}=2.25 \mathrm{~mA}$ (curve with rectangles) and $I_{C L}=-2.25 \mathrm{~mA}$ (curve with circles).

expressed more strongly than the regime of motion to the opposite direction.

\section{THEORETICAL RESULTS AND DISCUSSION}

To investigate the reasons leading to occurrence of such asymmetry, let us consider the sine-Gordon equation

$$
\phi_{t t}+\alpha \phi_{t}-\phi_{x x}=\beta \phi_{x x t}+\eta(x)-\eta_{c}(x) \sin \phi,
$$

where indices $t$ and $x$ denote temporal and spatial derivatives, respectively, $\phi$ is the Josephson phase difference. Space and time are normalized to the Josephson penetration length $\lambda_{J}$ and to the inverse plasma frequency $\omega_{p}^{-1}$, respectively, so the voltage is $v=V / V_{J}=2 \pi V /\left(\Phi_{0} \omega_{p}\right), \quad \alpha=\omega_{p} / \omega_{c}$ is the damping parameter, $\omega_{p}=\sqrt{2 e I_{c} / \hbar C}, \omega_{c}=2 e I_{c} R_{N} / \hbar$, and $\beta$ is the surface loss parameter. Here, $\eta(x)$ is the spatial distribution of dc overlap bias current density and $\eta_{c}(x)$ is the critical current density, both normalized to the critical current density $J_{c}$, i.e., $\eta(x)=\eta_{0} f(x)$ with $\frac{1}{L} \int_{0}^{L} f(x) d x=1, \frac{1}{L} \int_{0}^{L} \eta_{c}(x) d x=1$. The boundary conditions have the form

$$
\begin{aligned}
& \phi(0, t)_{x}+\beta \phi(0, t)_{x t}=\Gamma-\Delta \Gamma, \\
& \phi(L, t)_{x}+\beta \phi(L, t)_{x t}=\Gamma+\Delta \Gamma .
\end{aligned}
$$

Here, $L$ is the dimensionless length of the junction in units of $\lambda_{J}, \Gamma=H_{e} /\left(\lambda_{J} J_{c}\right)$ is the normalized magnetic field, and $\Delta \Gamma$ is a small magnetic-field difference. ${ }^{25}$ Since both overlap $\eta_{o v}=\eta(x)$ and inline $\eta_{i n}=2 \Delta \Gamma / L$ components of the current are present, the total current density $i$, with respect to which all current-voltage characteristics will be computed, is the sum of overlap and inline components. ${ }^{35}$ More general approach to account for the spatial dependence of the magnetic field requires inclusion of the additional term $-d \Gamma(x) / d x$ into the sine-Gordon equation ${ }^{36}$ that for linear dependence of $\Gamma(x)$ gives the same current-voltage characteristic as the sum of overlap and inline current components. ${ }^{25}$ While the use of the sine-Gordon equation for modelling of YBCO GBJ is still an open question, ${ }^{37}$ due to, e.g., nonsinusoidal current-phase relation, ${ }^{38}$ the detailed investigations of $I_{c}(\phi)$ for $24^{\circ}$ GBJs demonstrated its 
sinusoidal character, ${ }^{39}$ and for $32^{\circ}$ GBJs the considered model has been successfully used. ${ }^{31}$

The computer simulations of the sine-Gordon equation are performed for the following parameters: $\alpha=1.5$ and $\beta=0.1$, external magnetic field $\Gamma=1$ corresponding to the sharper flux-flow step, and junction length $L=200$.

The first reason, which can lead to the diversity of the flux-flow steps for opposite magnetic field directions, is the asymmetry of the magnetic field produced by the coil. We repeated our measurements in different setups with various sample holders and coils with diameters from $5 \mathrm{~mm}$ to $15 \mathrm{~mm}$ and observed the repetition of the described effect. Moreover, the numerical investigation shows that to achieve such an effect the magnetic-field asymmetry $2 \Delta \Gamma$ must be more than $80 \%$, which is an overestimation in comparison with realistic experimental values. Thus, the possible asymmetry of the external field can not lead to the observed effect. Also, we have studied possible field focusing effect, when the magnetic field is larger at the center of the junction than at its edges. To model it, following Ref. 36, we add into Eq. (1) the derivative of the magnetic field term $-d \Gamma(x) / d x$, where $\Gamma(x) \sim a \sin (\pi x / L)$, see. Refs. 2 and 40. Since the function $\Gamma(x)$ is symmetric with respect to the spatial coordinate $x$, and due to the fact that $d \Gamma(x) / d x$ changes the sign with the change of the magnetic field sign, it must not lead to asymmetry of the current-voltage characteristic, since fluxons feel the same inhomogeneity irrespectively of the magnetic field sign. Indeed, our simulations confirm that the curves for the opposite values of magnetic field agree with each other, and can not, therefore, explain the difference in the flux flow steps.

Another possible reason of asymmetric behavior for the opposite magnetic field direction is the nonuniform fluxon chain penetration due to the grain boundary defects. This results in the asymmetric critical current distribution with various features. ${ }^{38,41,42}$ To model this, we have considered profiles of $\eta_{c}(x)$ qualitatively similar to depicted in Fig. 6 of Ref. 15 (see the inset of Fig. 4) with the decay law

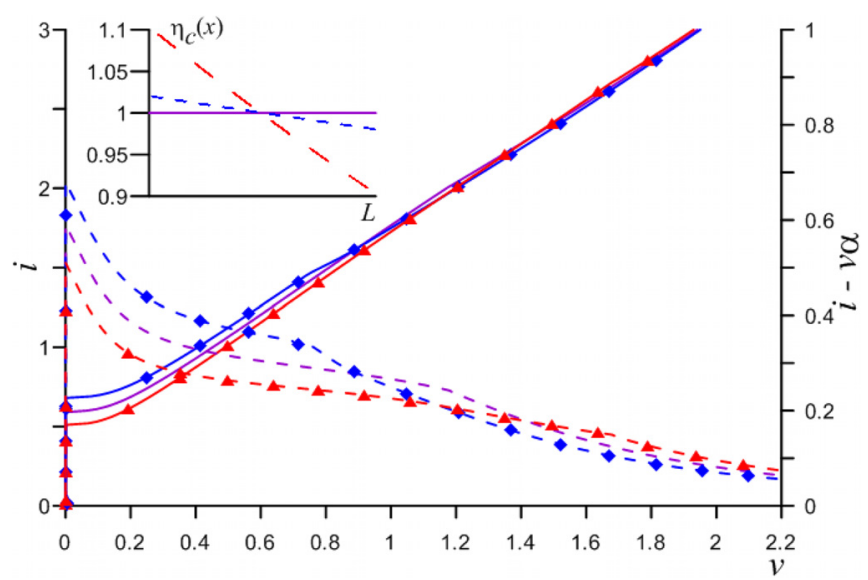

FIG. 4. IV characteristics $i$ vs $v$ (solid) and $(i-v \alpha)$ vs $v$ (dashed) for various critical current profiles $\eta_{c}(x) \sim \exp (-p x)$ : solid curve-uniform distribution $(p=0)$, curve with diamonds $(\Gamma=-1)$ and curve with triangles $(\Gamma=1)$ for $p=0.001$. The inset: current profiles with different decay factors: solid line-uniform distribution $(p=0)$, long dashed curve $-p=0.001$, and short dashed curve $-p=0.0002$. $\sim \exp (-p x)$. If we consider weak inhomogeneity of the critical current $(p<0.001)$, the flux flow steps are almost the same regardless of the field direction and are similar to the case of the uniform current distribution (solid line in the inset of Fig. 4). When we increase $p$ to the value of 0.001 , the fluxon penetration becomes asymmetric: for the positive $\Gamma$ (curve with triangles) fluxons start to move from the right end with the lower critical current and slow down their movement to the output left end where the critical current is larger. Thus, the fluxons are forced to enter and are stacked there in contrast to the case of negative $\Gamma$ (curve with diamonds) where the fluxons are effectively removed from the junction, which results in a much stronger flux-flow step. However, the critical current density asymmetry for $p=0.001$ leads to the asymmetric $i_{c}(\Gamma)$ characteristic, which is not observed in our experiment. In Fig. 5, the normalized experimentally obtained $i_{c}(\Gamma)$ curve is compared with the computer simulation results for various decay factors $p=0, p=0.001$ for $\eta_{c}(x)$, and $p=0.0002$ for $\eta(x)$. While the theoretical curves have the current decrease to almost zero for $\Gamma \approx 2 ;-2$, because our model does not account for a flux creep effect typical to the GBJ, the general behavior of the experimental curve qualitatively fits the case $p=0$. Thus, we can conclude that the asymmetric critical current distribution is unlikely to be the main reason of the observed asymmetry of the flux-flow steps.

The microstructural analysis of samples indicates the grain size $\sim 1 \mu \mathrm{m}$, so homogeneous bias current distribution along $\sim 350 \mu \mathrm{m}$ junction can be expected. However, GBJ anisotropy ${ }^{15}$ can lead to a redistribution of currents resulting in a certain bias current $\eta(x)$ asymmetry. To get the picture similar to the experimental one, we have performed the same analysis as for $\eta_{c}(x)$ (Fig. 4) and obtained the most appropriate decay factor $p=0.0002$ for $\eta(x) \sim \exp (-p x)$. In Fig. 6, two fluxflow steps for opposite field directions are shown. For the positive $\Gamma$ (curve with triangles), fluxons start to move from right end with the lower bias current and are accelerated under the Lorentz force action to the output (left) end. In the case of negative $\Gamma$ (curve with diamonds), the fluxons are forced to

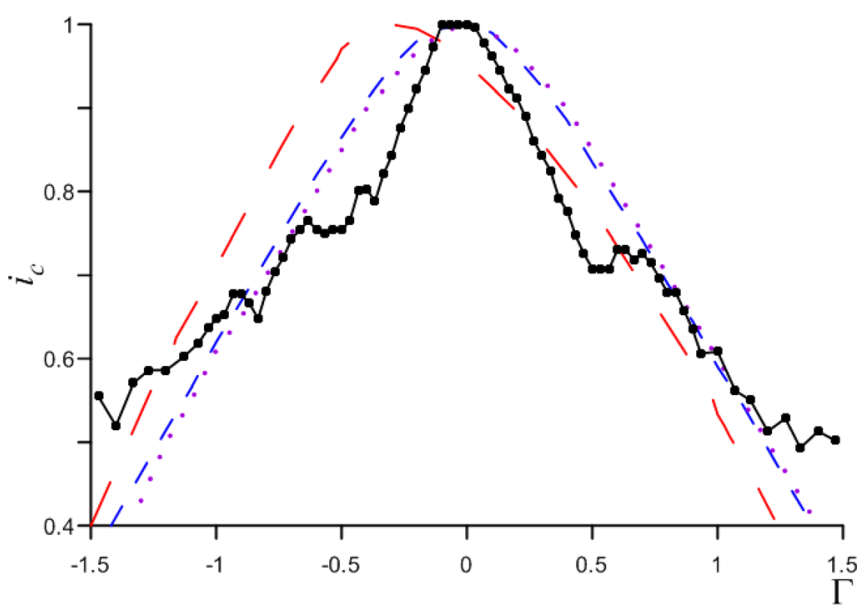

FIG. 5. Normalized critical current $i_{c}$ versus normalized magnetic field $\Gamma$ for experiment (solid curve with symbols) and theory with the symmetric $p=0$ (dotted curve), asymmetric critical current $p=0.001$ (long dashed curve), and asymmetric bias current $p=0.0002$ (short dashed curve). 


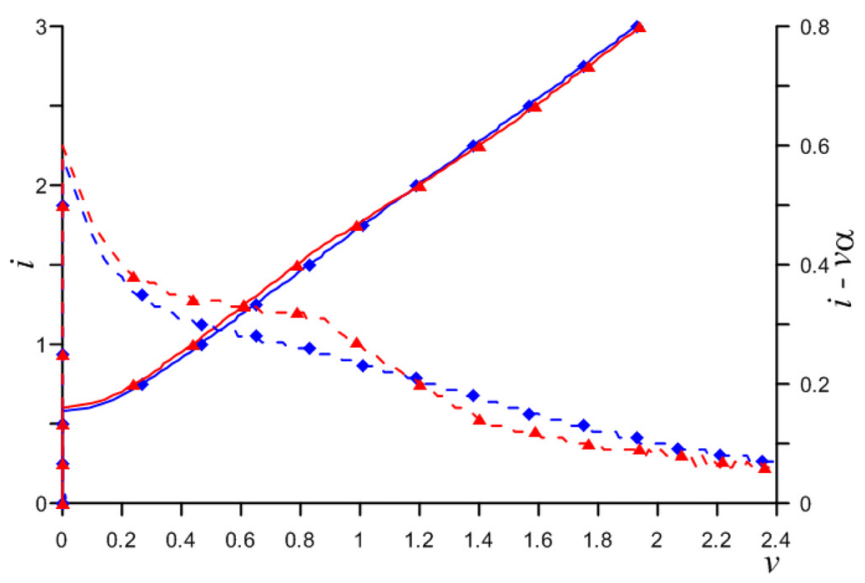

FIG. 6. $i$ vs $v$ (solid) and $(i-v \alpha)$ vs $v$ (dashed) for bias current profile $\eta(x) \sim \exp (-p x)$ with $p=0.0002$ : curve with diamonds $-\Gamma=-1$ and curve with triangles $-\Gamma=1$.

enter, but are not removed effectively. Here, $i_{c}(\Gamma)$ is nearly symmetric (see the short-dashed curve in Fig. 5), which is in agreement with our measurements. Taking into account that the magnitude of flux flow steps discrepancy is nearly the same for critical current asymmetry with $p=0.001$, Fig. 4, and bias current asymmetry with $p=0.0002$, Fig. 6 , this signals that the bias current asymmetry is the most probable reason, leading to the observed effect.

\section{CONCLUSIONS}

In conclusion, the current-voltage characteristics of long $24^{\circ}$ [001]-tilt $\mathrm{YBa}_{2} \mathrm{Cu}_{3} \mathrm{O}_{7-\delta}$ bicrystal grain-boundary junctions in the presence of magnetic field are studied experimentally and theoretically. The flux-flow steps with different height and slope depending on the external magnetic field direction are observed. The possible reasons leading to such asymmetry are investigated using the sine-Gordon equation. It is shown that the asymmetry of the bias current distribution ${ }^{15}$ is the most probable reason for the flux-flow steps difference.

\section{ACKNOWLEDGMENTS}

The authors wish to thank E. V. Il'ichev, M. Yu. Kupriyanov, and E. B. Goldobin for comments and discussions. The work was supported by Russian Ministry of Science (Project Nos. 2012-1.2. 1-12-000-1011-036/8401, 14.132.21.1431, and 11.G34.31.0029), Dynasty Foundation, Human Capital Foundation, and Russian Foundation of Basic Research (Project No. 12-08-01048).

${ }^{1}$ C. S. Owen and D. J. Scalapino, Phys. Rev. 164, 538 (1967).

${ }^{2}$ R. Monaco, M. Aaroe, J. Mygind, and V. P. Koshelets, J. Appl. Phys. 104, 023906 (2008).

${ }^{3}$ M. Moshe, V. G. Kogan, and R. G. Mints, Phys. Rev. B 79, 024505 (2009).

${ }^{4}$ S. Scharinger, C. Gürlich, R. G. Mints, M. Weides, H. Kohlstedt, E. Goldobin, D. Koelle, and R. Kleiner, Phys. Rev. B 81, 174535 (2010).
${ }^{5}$ D. Dimos, P. Chaudhari, and J. Mannhart, Phys. Rev. B 41, 4038 (1990).

${ }^{6}$ O. Nesher and E. N. Ribak, Appl. Phys. Lett. 71, 1249 (1997).

${ }^{7}$ R. Monaco, V. P. Koshelets, A. Mukhortova, and J. Mygind, Supercond. Sci. Technol. 26, 055021 (2013).

${ }^{8}$ D. Dimos, P. Chaudhari, J. Mannhart, and F. K. LeGoues, Phys. Rev. Lett. 61, 219 (1988).

${ }^{9}$ A. V. Bobyl, M. E. Gaevski, S. F. Karmanenko, I. A. Khrebtov, V. N. Leonov, D. V. Shantsev, V. A. Solov'ev, and R. A. Suris, Physica C 266, 33 (1996).

${ }^{10}$ K. K. Likharev, V. K. Semenov, O. V. Snigirev, and B. N. Todorov, IEEE Trans. Magn. 15, 420 (1979).

${ }^{11}$ T. Nagatsuma, K. Enpuku, K. Sueoka, K. Yoshida, and F. Irie, J. Appl. Phys. 58, 441 (1985).

${ }^{12}$ A. L. Pankratov, Phys. Rev. B 66, 134526 (2002).

${ }^{13}$ A. L. Pankratov, Appl. Phys. Lett. 92, 082504 (2008).

${ }^{14}$ E. A. Matrozova, L. S. Revin, and A. L. Pankratov, J. Appl. Phys. 112, 053905 (2012).

${ }^{15}$ M. Yu. Kupriyanov, M. M. Khapaev, Y. Y. Divin, and V. N. Gubankov, JETP Lett. 95, 289 (2012).

${ }^{16}$ V. P. Koshelets, A. Shchukin, I. L. Lapytskaya, and J. Mygind, Phys. Rev. B 51, 6536 (1995).

${ }^{17}$ A. V. Ustinov, H. Kohlstedt, and P. Henne, Phys. Rev. Lett. 77, 3617 (1996).

${ }^{18}$ V. P. Koshelets, S. V. Shitov, A. V. Shchukin, L. V. Filippenko, J. Mygind, and A. V. Ustinov, Phys. Rev. B 56, 5572 (1997).

${ }^{19}$ G. Carapella, N. Martucciello, and G. Costabile, Phys. Rev. B 66, 134531 (2002).

${ }^{20}$ C. Soriano, G. Costabile, and R. D. Parmentier, Supercond. Sci. Technol. 9, 578 (1996).

${ }^{21}$ M. Cirillo, N. Grønbech-Jensen, M. R. Samuelsen, M. Salerno, and G. V. Rinati, Phys. Rev. B 58, 12377 (1998).

${ }^{22}$ M. Salerno and M. R. Samuelsen, Phys. Rev. B 59, 14653 (1999).

${ }^{23}$ M. Jaworski, Phys. Rev. B 60, 7484 (1999).

${ }^{24} \mathrm{P}$. Cikmacs, M. Cirillo, V. Merlo, and R. Russo, IEEE Trans. Appl. Supercond. 11, 99 (2001).

${ }^{25}$ A. L. Pankratov, A. S. Sobolev, V. P. Koshelets, and J. Mygind, Phys. Rev. B 75, 184516 (2007).

${ }^{26}$ M. Jaworski, Supercond. Sci. Technol. 21, 065016 (2008).

${ }^{27}$ M. Jaworski, Phys. Rev. B 81, 224517 (2010).

${ }^{28}$ L. S. Revin and A. L. Pankratov, Phys. Rev. B 86, 054501 (2012).

${ }^{29}$ Y. M. Zhang, D. Winkler, P.-A. Nilsson, E. A. Stepantsov, and E. Wikborg, Extended Abstracts of ISEC'93, (1993) 4c-30, p. 268.

${ }^{30}$ D. Winkler, Y. M. Zhang, P. A. Nilsson, E. A. Stepantsov, and T. Claeson, Phys. Rev. Lett. 72, 1260 (1994).

${ }^{31}$ Y. M. Zhang, D. Winkler, P.-A. Nilsson, and T. Claeson, Phys. Rev. B 51, 8684 (1995)

${ }^{32}$ D. V. Masterov, S. A. Pavlov, A. E. Parafin, and Yu. N. Drozdov, Tech. Phys. 52, 1351 (2007).

${ }^{33}$ Yu. V. Kislinskii, P. V. Komissinski, K. Y. Constantinian, G. A. Ovsyannikov, T. Yu. Karminskaya, I. I. Soloviev, and V. K. Kornev, JETP 101, 494 (2005).

${ }^{34}$ H. Hilgenkamp and J. Mannhart, Rev. Mod. Phys. 74, 485 (2002).

${ }^{35}$ J. Mygind, V. P. Koshelets, M. Samuelsen, and A. S. Sobolev, IEEE Trans. Appl. Supercond. 15, 968 (2005).

${ }^{36}$ E. Goldobin, A. Sterck, and D. Koelle, Phys. Rev. E 63, 031111 (2001).

${ }^{37}$ F. Tafuri and J. R. Kirtley, Rep. Prog. Phys. 68, 2573 (2005).

${ }^{38}$ E. Il'ichev, V. Zakosarenko, R. P. J. IJsselsteijn, H. E. Hoenig, H.-G. Meyer, M. V. Fistul, and P. Mueller, Phys. Rev. B 59, 11502 (1999).

${ }^{39}$ A. A. Golubov, M. Yu. Kupriyanov, and E. Ilichev, Rev. Mod. Phys. 76, 411 (2004).

${ }^{40}$ R. Monaco, M. Aaroe, J. Mygind, and V. P. Koshelets, Phys. Rev. B 79, 144521 (2009).

${ }^{41}$ A. V. Bobyl, M. E. Gaevski, I. A. Khrebtov, S. G. Konnikov, D. V. Shantsev, V. A. Solov'ev, R. A. Suris, and A. D. Tkachenko, Physica C 247, 7 (1995).

${ }^{42}$ A. Sugimoto, T. Yamaguchi, and I. Iguchi, Physica C 357, 1473 (2001). 